Revue internationale de l'économie sociale

Recma

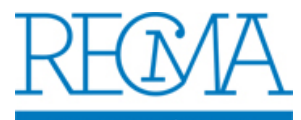

\title{
Quand les valeurs sont bousculées
}

\section{Jean-François Draperi}

Numéro 308, mai 2008

URI : https://id.erudit.org/iderudit/1021166ar

DOI : https://doi.org/10.7202/1021166ar

Aller au sommaire du numéro

Éditeur(s)

Association Recma

ISSN

1626-1682 (imprimé)

2261-2599 (numérique)

Découvrir la revue

Citer ce document

Draperi, J.-F. (2008). Quand les valeurs sont bousculées. Revue internationale de l'économie sociale, (308), 4-5. https://doi.org/10.7202/1021166ar d'utilisation que vous pouvez consulter en ligne.

https://apropos.erudit.org/fr/usagers/politique-dutilisation/ 


\section{QUAND LES VALEURS SONT BOUSCULÉES}

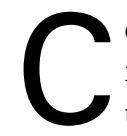

e numéro 308 de la Recma aborde sous des angles multiples la gouvernance des entreprises d'économie sociale. Quatre contributions présentées au colloque organisé par l'Istec (Ecole supérieure de commerce et de marketing), en collaboration avec l'Association internationale de management stratégique (AIMS), le Crédit mutuel et la Recma, se penchent sur des problèmes classiques de la gouvernance: la constitution d'un groupe hospitalier mutualiste qui adopte des stratégies de croissance externe, la gouvernance hybride - entre mutualisme et capitalisme - d'un groupe bancaire, la gestion du sociétariat dans une banque régionale, la relation entre efficacité économique et pratiques démocratiques. Ces contributions en sciences de gestion abordent des problématiques qui ne sont pas nécessairement spécifiques à l'économie sociale. Il n'en fallait pas moins pour évoquer les questions relativement nouvelles que les auteurs ambitionnent de traiter.

Au point de départ du travail de Jennifer Urasadettan, se trouve une clinique mutualiste qui, ayant acquis cinq cliniques privées, entreprend de "promouvoir l'esprit mutualiste " dans ces cliniques et d' " associer le personnel au changement de représentations et de pratiques ». Question essentielle en ces temps de croissance externe suivie par de toujours plus nombreuses coopératives et mutuelles, non seulement dans le secteur sanitaire et social, mais aussi et surtout dans les secteurs agricole, bancaire et assurantiel! Les résultats de l'enquête de Jennifer Urasadettan mettent en évidence un "couplage lâche " articulant la culture de chaque clinique et la culture mutualiste, couplage que requièrent des pratiques professionnelles quotidiennes adaptées à des environnements locaux.

Le groupe Crédit agricole est fréquemment cité dans les débats d'économie sociale, généralement au moment précis où l'on s'interroge sur les limites de celle-ci. Julien Batac, Vincent Maymo et Valérie Pallas-Saltiel éclairent utilement les termes de ce débat en soumettant à l'analyse les arguments habituellement avancés: l'évolution du couple produit-marché, le sociétaire et l'actionnaire, la croissance externe et son financement, le rapport à la Bourse. Ils concluent à l'émergence d'un nouveau modèle " hybride » autour de la notion de groupe, distinct à la fois de la société anonyme et de la société coopérative, modèle qui peut être comparé au groupe coopératif agro-alimentaire étudié par René Mauget (cf. Recma, $\mathrm{n}^{\text {os }} 278$, 279, 297).

Dominique Nouvel retrace l'itinéraire remarquable du Crédit mutuel Anjou de ses origines à nos jours, avant de se pencher sur la situation actuelle de la vie démocratique. Il souligne pour conclure l'importance de la "gestion du sociétariat " pour que le groupe résiste à la poursuite de la banalisation et au risque de démutualisation, gestion qui associe une dimension économique (qui fait du sociétaire un client des services bancaires) et une dimension politique (qui fait du sociétaire un militant de causes sociales). 
C'est précisément cette question qui est au cœur du travail de Fabrice Caudron. Au terme d'une discussion théorique très argumentée, l'auteur propose le concept de « rationalisation démocratique » pour désigner le processus commun de démocratisation et de rationalisation de la gestion. Ce terme permet de souligner que, loin d'être un état facilement identifiable, la démocratie est un processus toujours en mouvement. Il permet également de montrer que le processus de démocratisation n'est pas opposé à celui de rationalisation; au contraire, il indique une voie efficace pour intégrer une logique politique ou sociologique - telle qu'on la trouve dans les coopératives d'activités et d'emploi, par exemple, et plus largement dans les entreprises d'économie sociale - au sein du champ économique, logique qui questionne l'organisation fonctionnelle et technocratique.

Dans ce numéro, on lira également avec intérêt le texte de Christian Lion qui, à partir de l'étude de l'assurance mutuelle contre l'incendie entre 1815 et 1830, montre la proximité entre le secours mutuel et l'assurance mutuelle autour de la promotion d'un "certain type d'homme et de comportement ": un homme qui, en adhérant à la mutuelle, s'engage volontairement dans des relations solidaires, voire fraternelles. Cette réflexion frappe par son actualité, alors que l'économie sociale est confrontée à une redéfinition non juridique de ses contours.

Ricardo Abramovay attire notre attention sur l'essor significatif de nouvelles coopératives brésiliennes dans le secteur du crédit. Au-delà du service bancaire rendu, ces coopératives sont définies comme des "écoles d'entrepreneuriat collectif ", offrant à des personnes d'origine économique modeste la possibilité d'accéder à la responsabilité économique et, finalement, de s'émanciper.

Ces contributions - celles composant le dossier comme celles de Christian Lion et de Ricardo Abramovay - mettent en évidence la difficulté de comprendre la dynamique des entreprises d'économie sociale à partir d'un seul système de représentation et d'une seule forme d'organisation, définis a priori.

Jennifer Urasadettan, Julien Batac, Vincent Maymo, Valérie Pallas-Saltiel, Dominique Nouvel montrent, chacun à sa manière, les difficultés que rencontrent des entreprises d'une certaine taille et intervenant sur des marchés concurrentiels pour maintenir un modèle de fonctionnement compréhensible à partir des seules valeurs fondatrices de l'économie sociale. L'effort théorique de Fabrice Caudron constitue une tentative de dépassement de ces difficultés qui sont constatées quelle que soit la volonté des dirigeants: tout se passe comme si nous étions renvoyés à cet impératif de pragmatisme auquel se réfere si souvent l'acteur de l'économie sociale en prise avec un environnement toujours plus complexe. Au-delà du pragmatisme, les travaux composant ce numéro proposent de nouvelles lectures interprétatives dont nous ne doutons pas qu'elles susciteront l'intérêt et la critique des acteurs comme des chercheurs. 